\title{
Theoretical Analysis of Polarization Bistability in Vertical Cavity Surface Emitting Semiconductor Lasers
}

\author{
S. F. Yu
}

\begin{abstract}
The polarization bistability of vertical cavity surface emitting lasers under external optical injection is analyzed theoretically. It is found that on the condition where the optical gain is blue-shifted relative to the cavity mode, the bistable characteristics of VCSEL's can be improved significantly: 1) lowoptical power of the external injection light is required, 2) the influence of self-heating effects is minimized, and 3 ) wide bistable hysteresis loop width can be maintained. Furthermore, it is also shown that the reflectivity of Bragg reflectors should also be optimized to improve the performance of VCSEL's as bistable switches.
\end{abstract}

Index Terms - Optical bistability, optical polarization, semiconductor device modeling, semiconductor lasers, vertical cavity surface emitting lasers.

\section{INTRODUCTION}

$\mathbf{V}$ ERTICAL cavity surface emitting lasers (VCSEL's) have attractive features such as high-modulation bandwidth, low threshold current, single longitudinal mode operation with circular output beam profile and its feasibility to fabricate cost effective, large, dense two-dimensional arrays with wafer-scale processing and testing [1]. Extensive effort has been concentrated on the improvement of the threshold current and the transverse mode properties. On the other hand, the polarization control of VCSEL's is also crucial for their applications in polarization-sensitive equipment such as magneto-optic disks and coherent detection systems. Several methods have been proposed to stabilize the polarization of VCSEL's such as with antisotropic transverse cavity geometries [2] or anisotropic stress to the gain layer [3], [4]. The other approaches use polarization-sensitive Bragg reflectors [5] or metal-interlaced grating etched at the surface of Bragg reflector [6]. Some methods exhibit unreliable reproductivity of single polarization operation or others required extensive re-engineering of the device's structure. Alternatively, external optical injection can be utilized to stabilize the polarization of lasers. Furthermore, fast modulation between two orthogonal polarization states of VCSEL's has been reported by the external optical feedback of polarized light [7]. This implies that the utilization of external optical injection, VCSEL's have the potential applications in all-optical switching and optical memory.

Manuscript received October 31, 1996; revised March 10, 1997.

The author is with the Department of Electrical and Electronic Engineering, University of Hong Kong, Hong Kong.

Publisher Item Identifier S 0733-8724(97)04348-X.
Extensive studies have concentrated on the polarization bistability of Fabry-Perot devices under external optical injection [8] and it is expected that VCSEL's will also exhibit similar characteristics. However, the bistable mechanism of VCSEL's may exhibit differently to the Fabry-Perot devices due to 1) large gradient of quantum well gain spectrum contributes to the selection mechanism of orthogonal modes in VCSEL's and 2) optical gain is red-shifted due to the self-heating effects of VCSEL's. In this paper, the polarization bistability of VCSEL's is studied theoretically. A self-consistent rate-equation model with the consideration of different design of gain/cavity mode alignment, longitudinal distribution of optical fields as well as Bragg reflectivity to analyze the polarization bistability of VCSEL's. The influence of self-heating effects on the bistable mechanism of VCSEL's with external optical injection is also investigated.

\section{BASIC ASSUMPTIONS AND MODEL}

\section{A. Basic Assumptions}

In index guided VCSEL's with circular waveguide, the guided fundamental mode has no azimuthal variation and the optical field distribution is only dependent on the lateral position. Because there is no preferred axis of symmetry in the circular cross section and the electric field can be directed so that it is everywhere parallel to one of an arbitrary pair of orthogonal directions. In addition, the two orthogonal polarized lights receive same amount of optical gain due to the isotropic quantum well active layer such that the two orthogonal polarization states of VCSEL's are almost degenerated. However, in some situations the output light of the devices is linearly polarized along the $(011)$ or $(01 \overline{1})$ crystal direction [2], [9], [10]. This may be caused by the anisotropic stress applied on the active layer or strained active layer grown on misoriented substrate [4]. It is also shown the polarization gain-dependence is due to the spectral splitting between the polarization cavity-resonance such that it produces sufficient difference in gain to allow one polarization state to dominate [11]. In our model, the orthogonal TE polarization states are assumed to be polarized in the $X$ - and $Y$-directions which are parallel and perpendicular to the crystal plane (011), respectively. The $X$-polarized light is lasing for the VCSEL's biased slightly above threshold.

The dispersive bistability of VCSEL's is arisen from gain saturation which is similar to that of the Fabry-Perot devices. 
Gain saturation can be obtained through the input $Y$-polarized light as the optical gain is depleted in the process of amplifying the $Y$-polarized light, and thus $X$-polarized mode oscillation is suppressed. The reduction of carrier concentration which accompanies the amplification of $Y$-polarized mode also causes an increase in refractive index inside the active region. If the wavelength of the input $Y$-polarized light is set slightly longer than that of the $Y$-polarized light cavity wavelength without external optical injection, dispersive bistability is expected in both $X$ - and $Y$-polarization output [8] of VCSEL's. However, the dispersive bistability of VCSEL's may exhibit differently to that of Fabry-Perot devices due to the influence of self-heating effects. It is reported that mode-switching between $X$ - and $Y$-polarized modes can be achieved with the change of cavity temperature [11]. Hence, it is expected that the gain saturation power of $X$ - and $Y$-polarized modes as well as the dispersive bistability of VCSEL's are also temperature dependent. It must be noted that the distribution of optical power inside the VCSEL's is determined by the design of Bragg reflectors. This implies that the coupling strength between the input $Y$-polarized light and active region as well as the gain saturation power are also dependent on the devices' structure. Therefore, in order to analyze the polarization bistability characteristics of VCSEL's, the dispersive effects, the thermal effects and the structural effects have to be taken into consideration.

\section{B. Modeling of Wavelength Bistability in VCSEL's}

It is assumed that simultaneous $Y$-polarized light amplification takes place when a $Y$-polarized light is injected into a laser diode. The term of carrier consumption due to the $Y$-polarized light injection can be introduced into the rate equation of carrier concentration as

$$
\frac{\partial N}{\partial t}=\frac{J}{e L_{z} N_{w}}-\frac{N}{\tau_{N}}-\nu_{X} \Gamma_{X} G_{X} S_{X}-\nu_{Y} \Gamma_{Y} G_{Y} S_{Y}
$$

where $J$ is the injection current density, $e$ is the electron charge, $L_{z}$ is the width of quantum well and $N_{w}$ is the number of wells. $\tau_{N}$ is the carrier lifetime and $N$ is the carrier concentration inside the active layer. $S_{X(Y)}$ is the average photon density inside the active layer, $\Gamma_{X(Y)}$ is the confinement factor and $\nu_{X(Y)}$ is the group velocity of the two nearly-degenerate orthogonal fundamental TE polarization modes. The optical gain $G_{X(Y)}$ given in (1) is dependent on the temperature and the oscillation wavelength of $X$ - and $Y$ polarized light. The calculation of optical gain and its method of implementation in (1) can be found in the Appendix A.

The photon density of the $X$-polarized light can be described by

$$
\frac{\partial S_{X}}{\partial t}=\left(\nu_{X} \Gamma_{X} G_{X}-1 / \tau_{X}\right) S_{X}+K B_{\mathrm{sp}} N^{2}
$$

where $K$ is the spontaneous emission factor, $B_{\mathrm{sp}}$ is the bimolecular carrier recombination coefficient. The output power of $X$-polarized light from the right surface $P_{X \text { out }}$ can be expressed as

$$
P_{X o u t}=\frac{1}{2} \hbar \omega \pi w^{2} \Gamma_{Z} \Gamma_{x}^{-1}\left(1-r_{X R}^{2}\right) S_{X}
$$

where $\hbar$ is the Plank's constant, $r_{X R}$ is the facet reflectivity of the right surface of $X$-polarized light, $\Gamma_{Z}$ is the coupling factor and $w$ is the radius of the core region.

In addition, the influence of self-heating effects can be taken into calculation by [12]

$$
C_{\mathrm{th}} \frac{\partial T}{\partial t}=\left(P_{T V}+P_{Y \text { in }}-P_{\text {out }}\right)-\kappa \frac{\Delta T}{R_{\mathrm{th}}}
$$

where $C_{\text {th }}$ is defined as the thermal capacitance, $R_{\mathrm{th}}$ is the equivalent thermal resistor of VCSEL's, $\kappa$ is the thermal conductivity, $P_{Y \text { in }}$ is the power of input $Y$-polarized light, $P_{\text {OUT }}\left(=P_{X \text { out }}+P_{Y \text { out }}\right.$ ) is the total output power of the laser and $P_{T V}\left(=J \cdot \pi w^{2} \cdot V_{f}\right)$ is the total input electrical power. $V_{f}$ is the voltage across the active layer and is given by [12]

$$
\begin{gathered}
V_{f}=\frac{1}{q}\left[E_{g}+k_{B} T \cdot \log \left\{\left(\exp \left(N / N_{c}\right)-1\right)\right.\right. \\
\left.\cdot\left(\exp \left(N / N_{v}-1\right)\right\}\right]
\end{gathered}
$$

where $E_{g}\left(=1.519-4.408 \times 10^{-4}\left(T^{2} /(T+204)\right)\right.$ is the effective energy gap of the quantum well active layer and $k_{B}$ is the Boltzmann constant. $N_{c}$ and $N_{v}$ are the effective conduction and valence edge density of states, respectively.

The effective photon lifetime of the $X$-polarized light, $\tau_{X}$, given in (2) and the coupling factor, $\Gamma_{Z}$, given in (3) can be determined with the structural and material parameters of VCSEL's (i.e., dimension and refractive index of the dielectric layers) taken into calculation. This can be done by using the transfer matrix method to obtain the threshold condition of laser. The threshold condition requires the optical fields to reproduce itself after each round trip under steady state operating condition. This leads to the condition

$$
\begin{aligned}
& m x_{21} r_{X L} e^{2 \gamma_{X, 1} \Delta L_{1}}+m x_{22}-r_{X R} \\
& \cdot\left(m x_{11} r_{X L} e^{2 \gamma_{X, 1} \Delta L_{1}}+m x_{12}\right)=0
\end{aligned}
$$

where the derivation of (6) can be found in the Appendix B. Solving (6) gives the threshold gain, resonant frequency as well as the intensity distribution profile of $X$-polarized light. Therefore, $\tau_{X}$ and $\Gamma_{X}$ can also be evaluated from the threshold condition of VCSEL's.

In order to describe the dispersive bistability in VCSEL's, the wavelength of the injected $Y$-polarized light and its dependence on the Bragg reflectors have to be taken into consideration. Therefore, the matrix method is again utilized to describe the injected fields inside the cavity of VCSEL. Using the matrix method, the input $Y$-polarized propagation field, $F_{\text {in }}$, and output $Y$-polarized propagation field, $F_{\text {out }}$, are related by (7) at the bottom of the next page.

Furthermore, the $Y$-polarized fields distribution inside the laser cavity are given by

$$
\begin{aligned}
& F_{k}=\left(r_{Y L} e^{2 \gamma_{Y, 1} \Delta L_{1}} p_{11}+p_{12}\right) R_{1}+p_{11} t_{Y L} e^{\gamma_{Y, 1} \Delta L_{1}} F_{\text {in }} \\
& R_{k}=\left(r_{Y L} e^{2 \gamma_{Y, 1} \Delta L_{1}} p_{21}+p_{22}\right) R_{1}+p_{21} t_{Y L} e^{\gamma_{Y, 1} \Delta L_{1}} F_{\text {in }}
\end{aligned}
$$




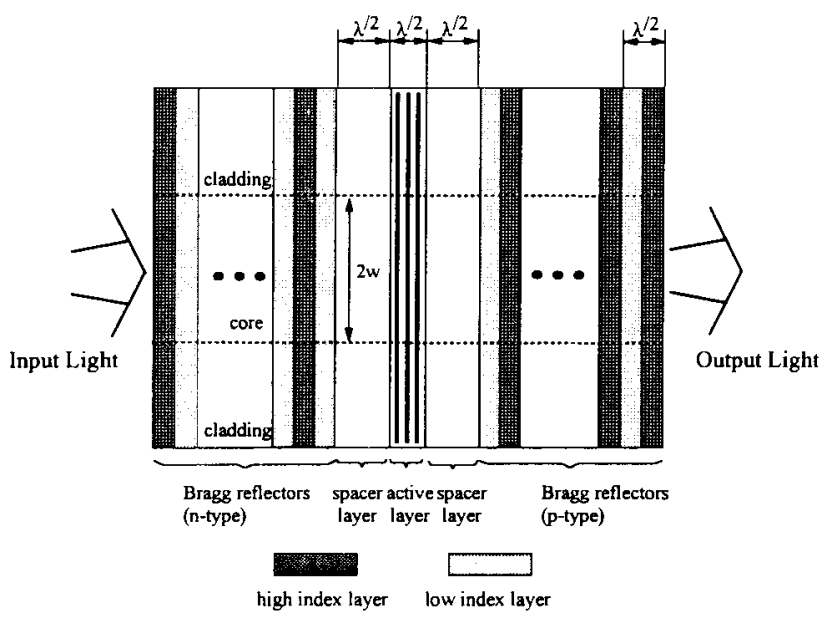

Fig. 1. Schematic of an index guided vertical cavity surface emitting laser used in our analysis.

where $k$ is an integer. The electric field $R_{1}$ given in (8) is expressed as

$$
\begin{aligned}
& \frac{R_{1}}{F_{\text {in }}}= \\
& \quad \frac{\left(m y_{11} r_{Y R}-m y_{21}\right) t_{Y L} e^{\gamma_{Y, 1} \Delta L_{1}}}{m y_{22}-r_{Y R} m y_{21}+\left(m y_{21}-r_{Y R} m y_{11}\right) r_{Y L} e^{2 \gamma_{Y, 1} \Delta L_{1}}} .
\end{aligned}
$$
C.

The derivation of (7), (8), and (9) are shown in the Appendix

The optical power of the input $Y$-polarized light, $P_{Y \text { in }}$, is defined as

$$
P_{Y \text { in }}=\frac{1}{2} \hbar \omega \pi w^{2} \Gamma_{Y}^{-1} S_{Y \text { in }}
$$

where $S_{Y \text { in }}$ is the injected photon density. The corresponding output power of $Y$-polarized light $P_{Y \text { out }}$ can be obtained from (7)

$$
P_{Y \text { out }}=\left(1-r_{Y L}^{2}\right)\left(\left|F_{\text {out }}\right|^{2} /\left|F_{\text {in }}\right|^{2}\right) P_{Y \text { in }} .
$$

The corresponding average photon density $S_{Y}$ inside the active layer is given by

$$
\begin{aligned}
S_{Y} / S_{Y \text { in }}= & \sum_{k}\left[\left(1+e^{-2 \operatorname{Re}\left[\gamma_{Y, k} \Delta L_{k}\right]}\right)\left|F_{k}\right|^{2}\right. \\
& \left.+\left(1+e^{2 \operatorname{Re}\left[\gamma_{\gamma, k} \Delta L_{k}\right]}\right)\left|R_{k}\right|^{2}\right] \Delta L_{k} /\left|F_{\text {in }}\right|^{2} L_{z} N_{w}
\end{aligned}
$$

where the right-hand side (RHS) of (12) represents the average photon power of $Y$-polarization inside the active layer. As the input power of the $Y$-polarized light and its wavelength are defined, the value of $S_{X}$ and $N$ can be solved self-consistently by using (1), (2), (4), and (12).
TABLE I

\begin{tabular}{l|l|l}
\hline Parameters & Symbol & Value \\
\hline \hline effective refractive index of activc layer (X-polarization) & $\mathrm{n}_{\mathrm{ax}}$ & 3.524 \\
\hline effective refractive index of active layer(Y-polarization) & $\mathrm{n}_{\mathrm{aY}}$ & 3.527 \\
\hline effective refractive index of spacer layer (both polarization) & $\mathrm{n}_{\mathrm{s}}$ & 3.393 \\
\hline effective refractive index of high index Bragg layer (both) & $\mathrm{n}_{\mathrm{h}}$ & 3.503 \\
\hline effective refractive index of low index Bragg layer (both) & $\mathrm{n}_{\mathrm{l}}$ & 2.950 \\
\hline
\end{tabular}

TABLE II

\begin{tabular}{l|l|l}
\hline Parameters & Symbol & Value \\
\hline \hline wavelength of X-polarized light (at threshold) & $\lambda_{\mathrm{X}}$ & $0.85 \mu \mathrm{m}$ \\
\hline wavelength of Y-polarized light (at threshold) & $\lambda_{\mathrm{Y}}$ & $0.85001 \mu \mathrm{m}$ \\
\hline group velocity of X-polarized light & $\nu_{\mathrm{X}}$ & $0.86 \times 10^{10} \mathrm{~cm} / \mathrm{s}$ \\
\hline group velocity of Y-polarized light & $\nu_{\mathrm{Y}}$ & $0.86 \times 10^{10} \mathrm{~cm} / \mathrm{s}$ \\
\hline confinement factor of X-polarized light & $\mathrm{I}_{\mathrm{X}}$ & 0.998 \\
\hline confinement factor of Y-polarized light & $\Gamma_{\mathrm{Y}}$ & 0.997 \\
\hline free carrier absorption \& scattering losses $(\mathrm{X}$-polarization) & $\alpha_{\mathrm{X}}$ & $20.0 \mathrm{~cm}$ \\
\hline free carrier absorption \& scattering losses (Y-polarization) & $\alpha_{\mathrm{Y}}$ & $20 . \mathrm{cm}^{-1}$ \\
\hline linewidth enhancement factor & $\alpha_{\mathrm{m}}$ & 3.0 \\
\hline differential gain coefficient & $\partial \mathrm{G} / \partial \mathrm{N}$ & $4 \times 10^{-16} \mathrm{~cm}^{2}$ \\
\hline temperature coefficient of refractive index & $\partial \mathrm{n} / \partial \Gamma$ & $1 \times 10^{-4} \mathrm{~K}^{-1}$ \\
\hline spontaneous emission factor & $\mathrm{K}$ & $1 \times 10^{-4}$ \\
\hline bimolecular carrier recombination coefficient & $\mathrm{B}_{\mathrm{sp}}$ & $1 \times 10^{-10} \mathrm{~cm}^{3} \mathrm{~s}$ \\
\hline carrier lifctime in active layer & $\tau_{\mathrm{N}}$ & $3 \times 10^{-9} \mathrm{~s}$ \\
\hline Thermal resistance & $\mathrm{R}_{\mathrm{ll}}$ & $1200 \mathrm{~cm}^{-1}$ \\
\hline thickness of quantum well & $\mathrm{L}_{\mathrm{Z}}$ & $100 \AA$ \\
\hline number of quantum well & $\mathrm{N}_{\mathrm{W}}$ & 3 \\
\hline radius of core region & $\mathrm{w}$ & $10 \mu \mathrm{m}$ \\
\hline
\end{tabular}

\section{RESULTS AND DisCUSSIONS}

\section{A. Laser Structure}

The schematic of VCSEL with a built-in index guided structure is shown in Fig. 1. The GaAs- $\mathrm{Al}_{0.3} \mathrm{Ga}_{0.7}$ As quantumwell active layer is sandwiched between two undoped spacer layers and two Bragg reflectors, which provide optical feedback for lasing. The undoped spacer layers have thickness of half-wavelength each and the Bragg reflectors are formed by alternating layers of AlAs and AlGaAs with quarterwavelength thickness dielectric layers on both the n- and p-side, respectively. The active region consists of three GaAs$\mathrm{Al}_{0.3} \mathrm{Ga}_{0.7}$ As quantum wells with well width of $100 \AA$ and the total thickness of the active layer is half-wavelength. This configuration of dielectric index layers (as shown in Fig. 1) is employed in our analysis for the reasons of maximum coupling between optical intensity and active layer as well as minimum threshold current.

Furthermore, it is assumed that the Bragg wavelength of the $X$-polarized light is equal to $0.85 \mu \mathrm{m}$ and the $\mathrm{Bragg}$ wavelength of the $Y$-polarized light is $0.1 \AA$ longer than $X$ polarized light such that these two polarization states are spec-

$$
\frac{F_{\text {out }}}{F_{\text {in }}}=\frac{t_{Y R} t_{Y L} e^{\gamma_{Y, 1} \Delta L_{1}}\left(m y_{11} m y_{22}-m y_{12} m y_{21}\right)}{m y_{22}+m y_{21} r_{Y L} e^{2 \gamma_{Y, 1} \Delta L_{1}}-r_{Y R}\left(m y_{12}+m y_{11} r_{Y L} e^{2 \gamma_{Y, 1} \Delta L_{1}}\right)} .
$$




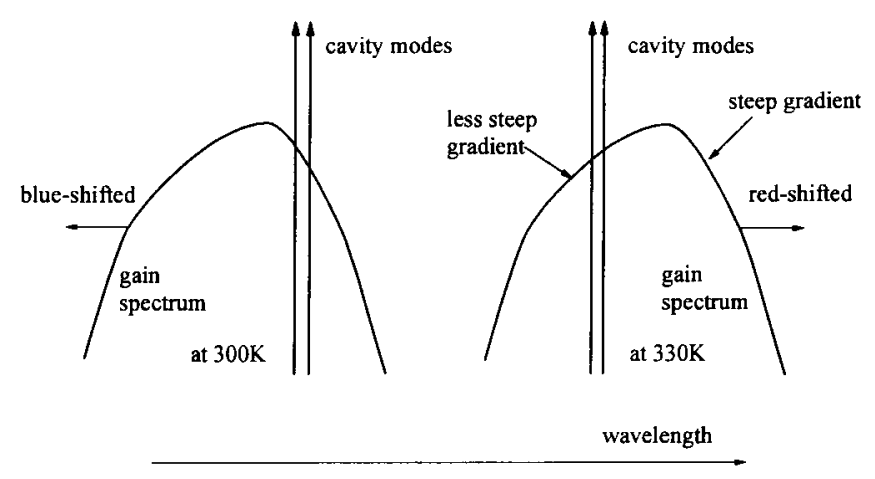

Fig. 2. Schematic for the alignment of optical gain spectrum and cavity modes of VCSEL's at background temperature of 300 and $330 \mathrm{~K}$. For the background temperature of $300 \mathrm{~K}$, the optical gain is blue-shifted while of $330 \mathrm{~K}$, the optical gain is red-shifted relative to the cavity mode.

trally nondegenerated due to polarization gain-dependence. For simple reasons, it is also assumed that the free carrier absorption loss is identical for both polarization light in all dielectric layers. The corresponding effective refractive index of the dielectric layers is given in Table I and other laser parameters used in the model are also given in Table II. In addition, to simplify our calculation, it is assumed that the fundamental TE polarization states are maintained by the builtin guided structure. In the following calculations, lasers are assumed to have symmetric Bragg reflectors on either sides of active layer and antireflection coated on both left and right surface (i.e., $r_{L}=r_{R}=0$ ). The number of dielectric layers of Bragg reflectors on the $n$ - and $p$ - sides are both equal to 42. The devices are biased at $1.1 \times$ its threshold current value and the $Y$-polarized light is injected into the left surface of the VCSEL's with wavelength detuned toward the longer Bragg wavelength by $1.1 \AA$.

\section{B. Influence of Gain/Cavity-Mode Alignment and Self-Heating Effects}

In VCSEL's, due to the spectral splitting of the $X$ - and $Y$ polarization modes, under the condition of blue-shifted gain, the shorter wavelength mode will experience enhanced gain overlap, while for red-shifted gain, longer wavelength mode will dominate such that switching of polarization modes is possible through the thermal effects as the optical gain spectrum can be red- or blue-shifted dependent on the cavity temperature [11]; see Fig. 2. Therefore, in the design of VCSEL's for polarization bistable operation, the choice of Bragg wavelength is critical due to the high thermal resistance of VCSEL's. In this section, we analyze the bistable characteristics of VCSEL's with optical gain blue- and red- shifted relative to the Bragg wavelength. This can be done by considering that the operating temperature of the device is at 300 and $330 \mathrm{~K}$ but keeping the cavity mode of the VCSEL's fixed at $0.85001 \mu \mathrm{m}$ (see Fig. 2). At $300 \mathrm{~K}$ the gain peak wavelength is 0.84407 $\mu \mathrm{m}$ and at $330 \mathrm{~K}$ the gain peak wavelength is $0.851573 \mu \mathrm{m}$ such that polarization switching is avoided for temperature rise less than $15 \mathrm{~K}$ inside the laser cavity.

Fig. 3 illustrates the bistability characteristics of VCSEL's with different thermal conductivity $\kappa(\mathrm{A}: \kappa=\infty, \mathrm{B}: \kappa=$
1.0, $\mathrm{C}: \kappa=0.9$ and $\left.\mathrm{D}: \kappa=0.45 \mathrm{~W} \mathrm{~cm}^{-1} \mathrm{C}^{-1}\right)$. The background temperature of the devices is set to $300 \mathrm{~K}$, and it is expected that temperature rise inside the laser cavity is due to the absorption of input $Y$-polarized optical power. Fig. (3a) and $(3 b)$ shows the output power ( $X$ - and $Y$ - polarization light) as a function of input $Y$-polarization light. It can be seen that the output $X$ - and $Y$-polarization light exhibit bistability with the input $Y$-polarization light. In general, the hysteresis loop becomes narrower with the decrease of $\kappa$ and is started to shift toward the left-hand side (low power side of $Y$-polarized light) as $\kappa$ is less than $0.9 \mathrm{~W} \mathrm{~cm}{ }^{-1} \mathrm{C}^{-1}$. But the hysteresis loop vanishes for $\kappa$ less than $0.2 \mathrm{~W} \mathrm{~cm}^{-1} \mathrm{C}^{-1}$.

Fig. (3c) illustrates the carrier concentration as a function of $Y$-polarization input power. When the $Y$-polarized light is injected and increased by a small amount, for the case "A," carrier concentration is clamped at the level for stimulated emission of $X$-polarized light. However, for the cases $\mathrm{B}$, $\mathrm{C}$, and $\mathrm{D}$, carrier concentration is slightly reduced with the input $Y$-polarized light but still weakly clamped at the upper level. This is due to the red-shifted of optical gain spectrum with the increase of cavity temperature which enhances the optical gain of the $X$-polarization light. Sudden drop of carrier concentration from its clamped level is observed for further increase of input $Y$-polarization light. The refractive index change induced by the carrier concentration would result in a shift of the Bragg wavelength, which will increase the mirror loss and destroy the condition of laser emission. As a consequence, the $X$-polarized light reduces to spontaneous emission, instead, the $Y$-polarized light amplification takes place. Fig. (3d) shows the change of devices' temperature under the influence of input $Y$-polarized light. Temperature increases linearly with the input power and kinks are observed (instead of hysteresis loops) at the point $X$-polarized light is quenched lasing.

Fig. 4 illustrates the bistability characteristics of VCSEL's with different thermal conductivity $\kappa(\mathrm{A}: \kappa=\infty, \mathrm{B}: \kappa=$ $0.9, \mathrm{C}: \kappa=0.2$, and $\left.\mathrm{D}: \kappa=0.1 \mathrm{~W} \mathrm{~cm}^{-1} \mathrm{C}^{-1}\right)$. The background temperature of the devices is set to $330 \mathrm{~K}$. Fig. 4(a) and (b) shows the output power ( $X$ - and $Y$-polarization light) as a function of injection power of input $Y$-polarization light. It can be seen that the output $X$-and $Y$-polarization light exhibit bistability with the input $Y$-polarization light. In general, the hysteresis loop becomes narrower with the decrease of $\kappa$ and is started to shift toward the right-hand side (high power side of $Y$-polarized light) as $\kappa$ is less than $0.9 \mathrm{~W} \mathrm{~cm}^{-1} \mathrm{C}^{-1}$.

Fig. (4c) illustrates the carrier concentration as a function of $Y$-polarization input power. In case A, the carrier concentration is clamped in the level of laser emission for the $X$ polarized light but slightly increase for cases B, C, and D (still weakly clamped at the upper level) by a small amount increase of input $Y$-polarized light. This is because the optical gain profile is red-shifted with the increase of cavity temperature such that the amount of carrier concentration is increased for the maintenance of optical gain level. Sudden drop of carrier concentration from its clamped level is observed for further increase of input $Y$-polarization light. Fig. (4d) shows the change of devices' temperature under the influence of input $Y$ polarized light. Temperature increases linearly with the input 


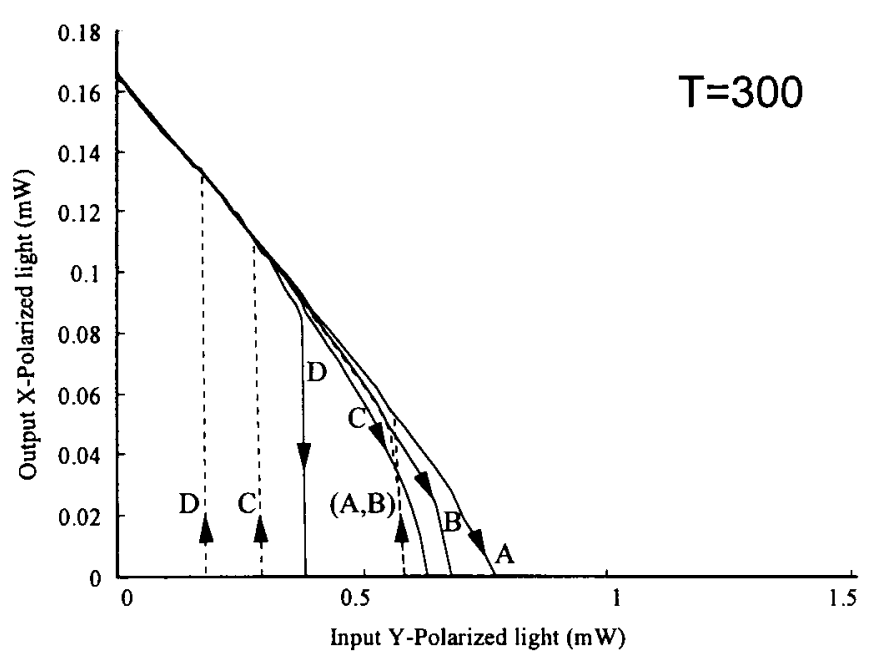

(a)

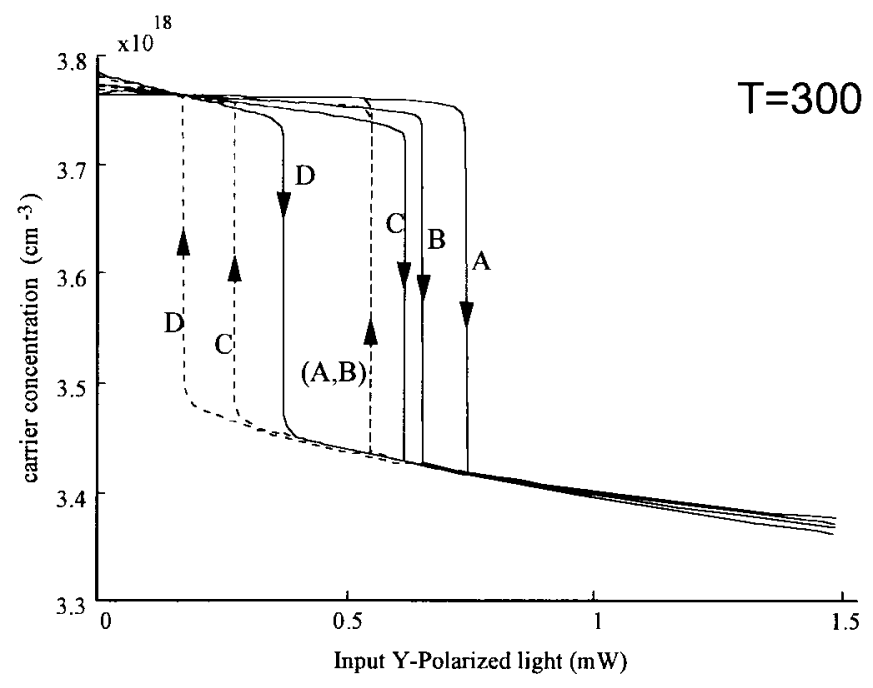

(c)

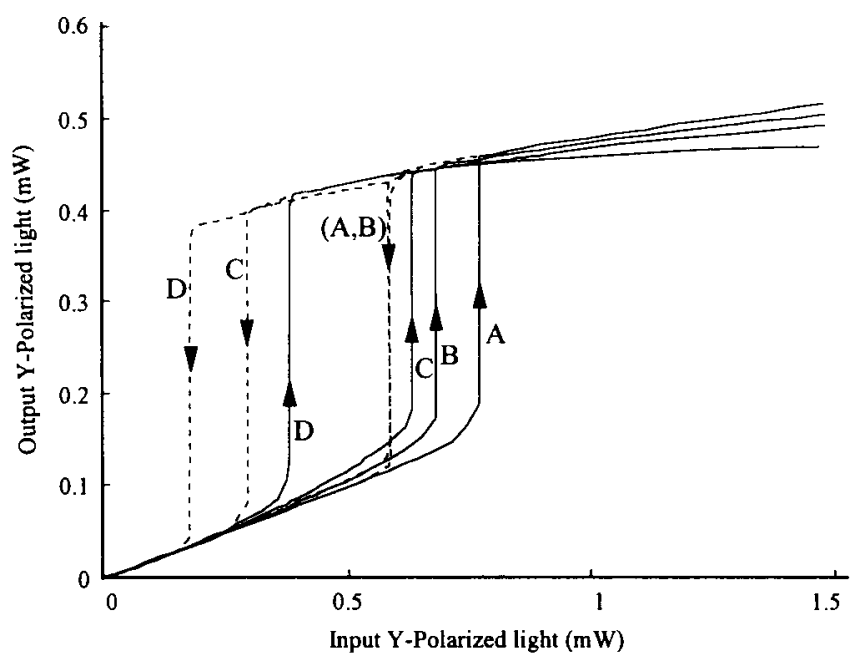

(b)

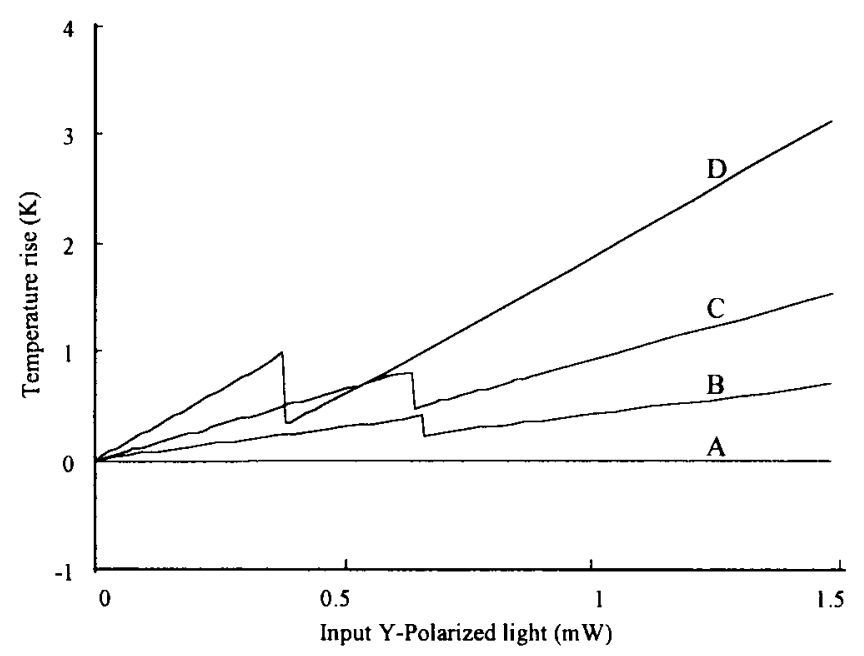

(d)

Fig. 3. The steady state characteristics of VCSEL with background temperature of $300 \mathrm{~K}$. (a) $X$-polarized light output versus $Y$-polarized light input, (b) $Y$-polarized light output versus $Y$-polarized light input, (c) carrier concentration versus $Y$-polarized light input, and (d) temperature rise versus input $Y$-polarized light input. Solid line-forward process and dashed line-reverse process.

power and kinks are observed (instead of hysteresis loops) at the point $X$-polarized light is quenched lasing.

The influence of cavity mode/gain alignment as well as selfheating effects on the polarization bistability of VCSEL's are compared and summarized as follows.

1) The hysteresis loops shift toward left- or right-hand side on the input/output plots (see Figs. 3 and 4) are dependent on the alignment between the optical gain and cavity mode. For the gain which is blue-shifted relative to the cavity mode, hysteresis loops exhibit left-shifted to low input power side. However, the opposite is observed for the red-shifted gain design.

2) Low-power dissipation in the blue-shifted gain design is obtained. This is because low input power is required for bistable operation such that the influence of self-heating effects at the active region can be minimized.

3) The thermal conductivity (as well as thermal resistivity) of VCSEL's should be confined at some range of magnitude for achieving polarization bistability.
Therefore, in order to utilize VCSEL's as optical switch or optical memory, it is required the gain should be blue-shifted relative to the cavity mode for the reasons of low input power and less heat dissipation inside the laser cavity.

The influence of input wavelength of $Y$-polarized light on the bistable mechanism of VCSEL's is also analyzed. We consider the case where the laser has $\kappa=0.9 \mathrm{~W} \mathrm{~cm}^{-1} \mathrm{C}^{-1}$ and background temperature is set to $300 \mathrm{~K}$. It can be shown VCSEL's demonstrate polarization bistability for the wavelength detuned toward the longer Bragg wavelength by not more than $9 \AA$; however, no polarization bistability is recorded for the wavelength detuned toward the shorter Bragg wavelength by more than $1 \AA$. Similar behavior of VCSEL's is observed at the background temperature of $330 \mathrm{~K}$.

\section{Influence of Bragg Reflectivity}

The longitudinal distribution of optical field inside the VCSEL's is dependent on the design of Bragg reflectors. In order to obtain polarization bistability in VCSEL's, it is 


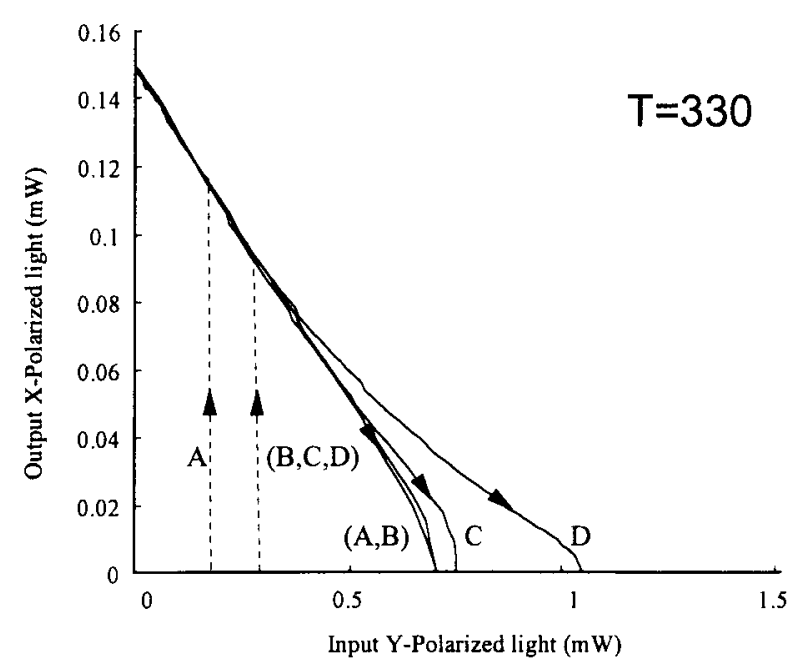

(a)

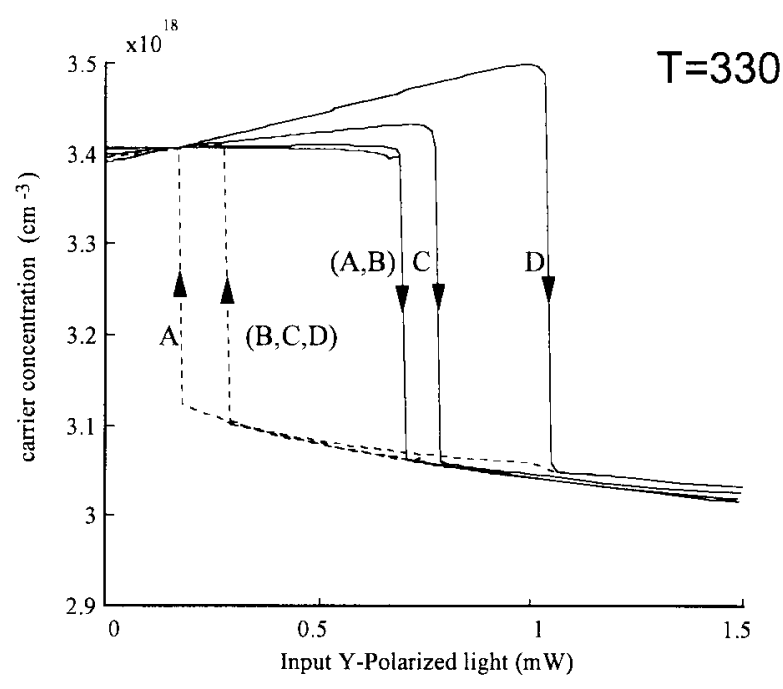

(c)

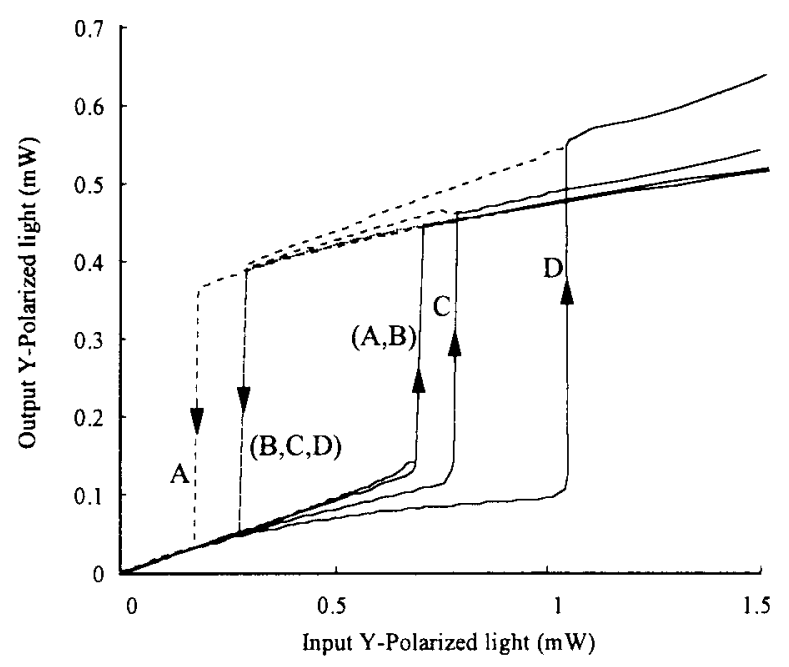

(b)

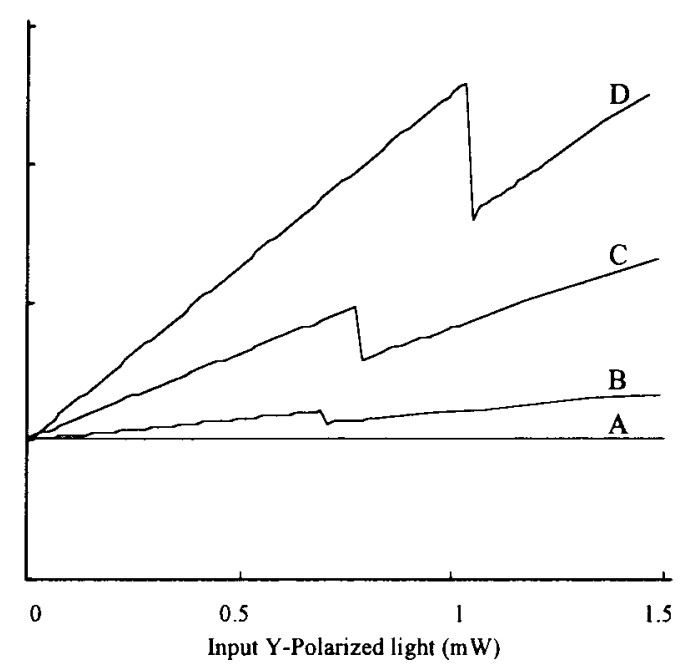

(d)

Fig. 4. The steady state characteristics of VCSEL with background temperature of $330 \mathrm{~K}$. (a) $X$-polarized light output versus $Y$-polarized light input and (b) $Y$-polarized light output versus $Y$-polarized light input. (c) Carrier concentration versus $Y$-polarized light input and (d) temperature rise versus input $Y$-polarized light input. Solid line-forward process and dashed line-reverse process.

required to consider the influence of Bragg reflectors on the coupling strength between the external injection light and active layer for reasonable injection power and hysteresis loops width. In this section, the influence of Bragg reflectivity on the bistable mechanism of VCSEL's is studied. The parameters used are the same as Fig. 3 except $\kappa=1 \mathrm{~W} \mathrm{~cm}^{-1} \mathrm{C}^{-1}$ and the number of layers of Bragg reflectors on both $n$ - and $p$-side are varied between A: 46, B: 42, and C: 38 . Fig. 5 shows the bistable characteristics of VCSEL's with different number of dielectric layers. As we can see, width of hysteresis loops is increased with the number of dielectric layers; however, high input optical power is required. This is because the increase in number of dielectric layers enhance the reflectivity of Bragg reflectors such that high external optical injection is required. On the other hand, increase in number of dielectric layers enhance the coupling strength between the injected optical power and active layer such that the wide hysteresis loops is obtained. The above analysis is also repeated for devices with background temperature of $330 \mathrm{~K}$ and similar characteristics are observed.

\section{CONCLUSION}

The influence of spectral mismatch between the gain and cavity mode, self-heating effects as well as the Bragg reflectivity on the polarization bistability of VCSEL's is studied. It is found that 1) input power required for achieving polarization bistability is dependent on the alignment between gain and cavity mode. When the gain is blue-shifted relative to the cavity mode, the hysteresis loops shift toward the low power side of input $Y$-polarized light especially for devices with low $\kappa$. However, the opposite is observed for the red-shifted alignment. 2) The influence of self-heating is minimized for the blue-shifted alignment due to low injection power is required for the bistable operation. 3) The reflectivity of Bragg reflectors affects the bistable characteristics of VCSEL's. For devices with low reflectivity (by reducing the number 


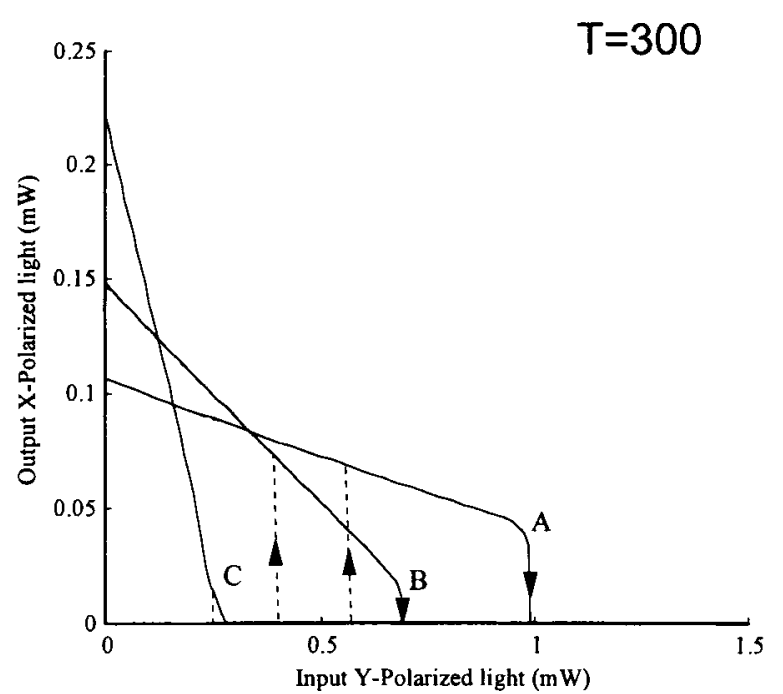

(a)

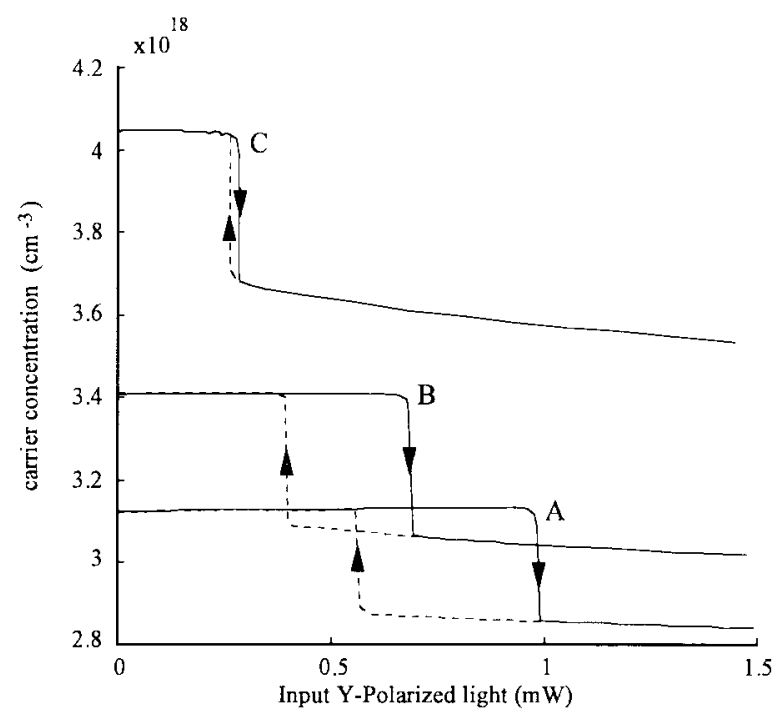

(c)

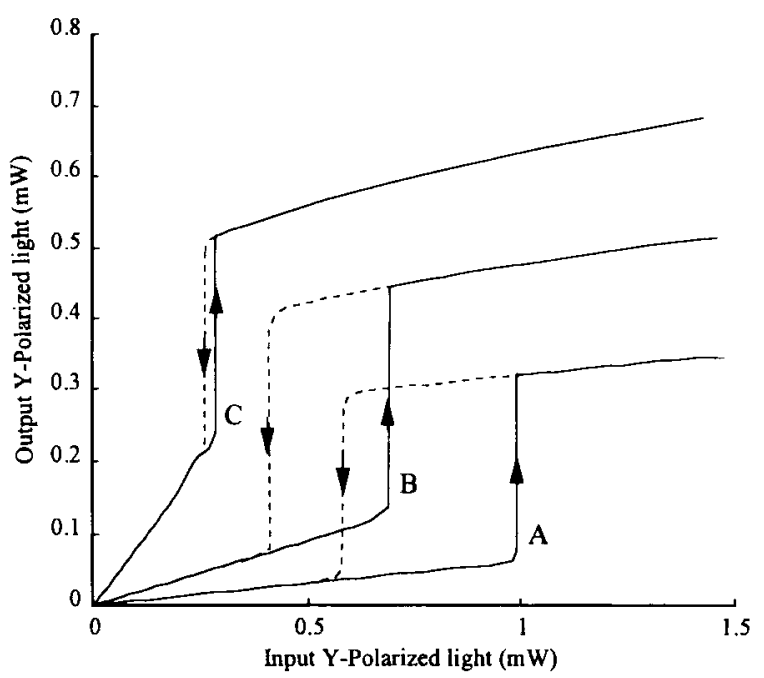

(b)

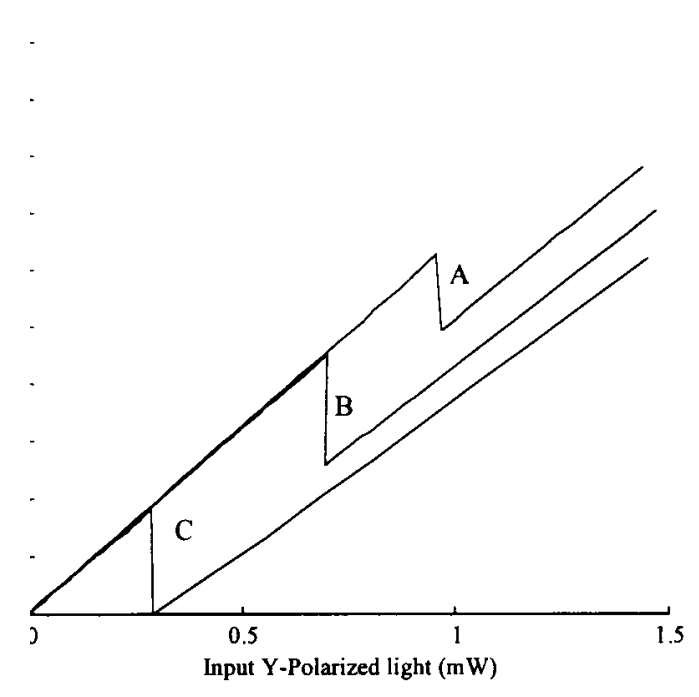

(d)

Fig. 5. The steady state characteristics of VCSEL with background temperature of $300 \mathrm{~K}$. (a) $X$-polarized light output versus $Y$-polarized light input and (b) $Y$-polarized light output versus $Y$-polarized. (c) Carrier concentration versus $Y$-polarized light input and (d) temperature rise versus input $Y$-polarized light input. Solid line-forward process and dashed line-reverse process.

of dielectric layers of Bragg reflectors), low input power is required to achieve bistable mechanism but the coupling strength between the injected light and active layer is reduced (i.e. narrow hysteresis loops). On the other hand, for VCSEL's with high reflectivity, coupling strength is improved but at the expense of low external injection power.

Therefore, in order to utilize VCSEL's for the application in all-optical switching and optical memory, it is recommended that the gain should be blue-shifted relative to the cavity mode for low input power requirement and less influence of selfheating effects. Furthermore, reflectivity of Bragg reflectors should also be optimized for suitable hysteresis loops width. In conclusions, a rate-equation model is developed with the consideration of gain/cavity mode alignment, distribution of longitudinal optical power and self-heating effects, to analyze the polarization bistability of VCSEL's under external optical injection. It is shown that all-optical switching and memory operation can be achieved in VCSEL's by external optical injection; however, the alignment of optical gain/cavity mode as well as Bragg reflection should be taken into consideration in order to minimize the requirement of high input power, to avoid self-heating effects as well as high tolerance of input power level (i.e., wide hysteresis loop width).

\section{APPENDIX A}

The gain spectrum of $\mathrm{GaAs} / \mathrm{Al}_{0.3} \mathrm{Ga}_{0.7} \mathrm{As}$ quantum wells' active layer can be obtained by using the $k \cdot p$ method and the detailed calculation can be found in [13]. However, direct implementation of the calculated data into our model required a huge database which is not practicable. Therefore, the optical gain is approximated as follows.

1) The dependence of peak gain on the injection carrier concentration and temperature is approximated by

$$
G_{p}=a_{N} \log \left(N / N_{o}\right)
$$




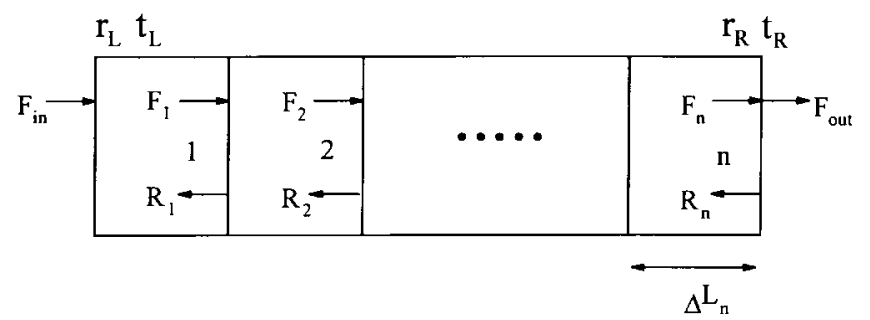

Fig. 6. Schematic of a multi-dielectric layers optical model for vertical cavity surface emitting laser: $F_{n}$ and $R_{n}$ is the forward and reverse field, respectively, and $\Delta L_{n}$ is the thickness of the $n$th dielectric layer. $F_{\text {in }}$ is the injection $F_{\text {out }}$ is the output field. $r_{L}$ and $t_{L}\left(r_{R}\right.$ and $\left.t_{R}\right)$ is the reflection and transmission coefficients on the left (right) surface.

where the temperature dependence of $a_{N}$ and $N_{O}$ can be expressed as

$$
\begin{aligned}
& a_{N}(T)=a_{0}+a_{1} T+a_{2} T^{2} \\
& N_{o}(T)=b_{0}+b_{1} T
\end{aligned}
$$

where $a_{0}=2478.3 \mathrm{~cm}^{-1}, a_{1}=-7.6369 \mathrm{~cm}^{-1} \mathrm{~K}^{-1}$, $a_{2}=1.3177 \times 10^{-2} \mathrm{~cm}^{-1} \mathrm{~K}^{-2}, b_{0}=8.4226 \times$ $10^{17} \mathrm{~cm}^{-3}$ and $b_{1}=5.0679 \times 10^{15} \mathrm{~cm}^{-3} \mathrm{~K}^{-1}$.

2) For the injection carrier concentration large than $3 \times 10^{18} \mathrm{~cm}^{-3}$, the gain peak is almost independent on the injection concentration. Hence, the variation of gain peak wavelength, $\lambda_{p}$, with temperature can be approximated by

$$
\lambda_{p}=\lambda_{0}+\lambda_{1} T
$$

where $\lambda_{0}=0.76904 \mu \mathrm{m}$ and $\lambda_{1}=2.501 \times 10^{-4}$ $\mu \mathrm{m} \mathrm{K}^{-1}$.

3) If the operation wavelength of the VCSEL's $\lambda_{X(Y)}$ is shifted less than or equal to $\pm 10 \mathrm{~nm}$ away from the gain peak, the gain spectrum can be approximated by

$$
G_{X(Y)}=G_{P}-G_{0}\left(\lambda_{X(Y)}-\lambda_{p}\right)^{2}
$$

where $G_{0}$ is a constant and is equal to $6.25 \times 10^{6} \mathrm{~cm}^{-1}$ $\mu \mathrm{m}^{-2}$ for $\lambda_{X(Y)}>\lambda_{p}$ but equal to $1.254 \times 10^{6} \mathrm{~cm}$ ${ }^{-1} \mu \mathrm{m}^{-2}$ for $\lambda_{X(Y)}<\lambda_{p}$ in order to take into account of the asymmetric gain profile.

\section{APPENDIX B}

The forward and reverse propagation fields of the $X$ polarized light, $F_{n}$, and $R_{n}$ at the right-hand surface can be expressed as (see Fig. 6) [14]

$$
\begin{aligned}
{\left[\begin{array}{l}
F_{n} \\
R_{n}
\end{array}\right]=} & \prod_{j=1}^{n-1}\left[\begin{array}{cc}
e^{\gamma X, j+1} \Delta L_{j+1} & 0 \\
0 & e^{-\gamma X, j+1} \Delta L_{j+1}
\end{array}\right] \\
& \cdot\left[\begin{array}{ll}
\frac{n x_{j+1}+n x_{j}}{2 n x_{j+1}} & \frac{n x_{j+1}-n x_{j}}{2 n x_{j+1}} \\
\frac{n x_{j+1}-n x_{j}}{2 n x_{j+1}} & \frac{n x_{j+1}+n x_{j}}{2 n x_{j+1}}
\end{array}\right]\left[\begin{array}{l}
F_{1} \\
R_{1}
\end{array}\right] \\
= & {\left[\begin{array}{ll}
m x_{11} & m x_{12} \\
m x_{21} & m x_{22}
\end{array}\right]\left[\begin{array}{l}
F_{1} \\
R_{1}
\end{array}\right] }
\end{aligned}
$$

where $F_{1}$ and $R_{1}$ are the propagation fields at the interface between first and second matrix section. $n x_{j(j+1)}$ is the effective refractive index of the $j$ th $(j+1$ th) dielectric layer. $\Delta L_{j+1}$ is the thickness of the $j+1$ th dielectric layer and $\gamma_{X, j+1}$ is defined as

$$
\gamma_{X, j+1}=\left\{\begin{array}{c}
\frac{1}{2}\left(\Gamma_{X} G_{X, j+1}-\alpha_{X}\right)+i 2 \pi n x_{j+1} / \lambda_{X} \\
\text { active layer } \\
-\frac{1}{2} \alpha_{X}+i 2 \pi n x_{j+1} / \lambda_{X} \\
\text { elsewhere }
\end{array}\right.
$$

where $i=\sqrt{-1}, c$ is the velocity of light in free space, $\lambda_{X}$ is oscillation wavelength of the lasing mode and $\alpha_{X}$ is the free carrier absorption loss of $X$-polarized light. The boundary conditions for the $X$-polarized light at the right and left surfaces are given as

$$
R_{n}=r_{X R} F_{n} \quad \text { and } \quad F_{1}=x_{X L} e^{2 \gamma_{X, 1} \Delta L_{1}} R_{1}
$$

where $r_{X R}$ and $r_{X L}$ is the reflectivity at right and left surface, respectively. Therefore, using (B1) and (B3), the eigenvalue equations for $F_{n}$ and $R_{1}$ can be obtained and is given by

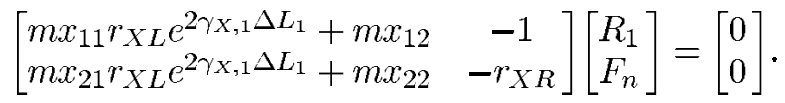

The threshold gain and the oscillation frequency of VCSEL can be determined from the root of the determinant of (B4) which is

$$
\begin{aligned}
& m x_{21} r_{X L} e^{2 \gamma_{X, 1} \Delta L_{1}}+m x_{22}-r_{X R} \\
& \cdot\left(m x_{11} r_{X L} e^{2 \gamma_{X, 1} \Delta L_{1}}+m x_{12}\right)=0
\end{aligned}
$$

such that the value of effective photon lifetime $\tau_{X}$ and the coupling factor $\Gamma_{Z}$ can also be calculated.

\section{APPENDIX C}

The forward and reverse propagation fields, $F_{n}$ and $R_{n}$ at the right hand surface can be expressed as (see Fig. 6) [14]

$$
\begin{aligned}
{\left[\begin{array}{l}
F_{n} \\
R_{n}
\end{array}\right]=} & \prod_{j=1}^{n-1}\left[\begin{array}{cc}
e^{\gamma, j+1} \Delta L_{j+1} & 0 \\
0 & e^{-\gamma Y, j+1} \Delta L_{j+1}
\end{array}\right] \\
& \cdot\left[\begin{array}{ll}
\frac{n y_{j+1}+n y_{j}}{2 n y_{j+1}} & \frac{n y_{j+1}-n y_{j}}{2 n y_{j+1}} \\
\frac{n y_{j+1}-n y_{j}}{2 n y_{j+1}} & \frac{n y_{j+1}+n y_{j}}{2 n y_{j+1}}
\end{array}\right]\left[\begin{array}{l}
F_{1} \\
R_{1}
\end{array}\right] \\
= & {\left[\begin{array}{ll}
m y_{11} & m y_{12} \\
m y_{21} & m y_{22}
\end{array}\right]\left[\begin{array}{l}
F_{1} \\
R_{1}
\end{array}\right] }
\end{aligned}
$$

where $n y_{j(j+1)}$ is the effective refractive index of the $j$ th $\left(j+1\right.$ th) dielectric layer. $\Delta L_{j+1}$ is the thickness of the $j+1$ th dielectric layer and $\gamma_{Y, j+1}$ is defined as

$$
\gamma_{Y, j+1}=\left\{\begin{array}{c}
\frac{1}{2}\left(\Gamma_{Y} G_{Y, j+1}-\alpha_{Y}\right)+2 \pi n y_{j+1} / \lambda_{Y} \\
\text { active layer } \\
-\frac{1}{2} \alpha_{Y}+i 2 \pi n y_{j+1} / \lambda_{Y} \\
\text { elsewhere }
\end{array}\right.
$$

where $i=\sqrt{-1}, c$ is the velocity of light in free space, $\lambda_{Y}$ is optical wavelength of the injection light and $\alpha_{Y}$ is the free carrier absorption loss of $Y$-polarized light. Inside the active 


$$
\frac{F_{\text {out }}}{F_{\text {in }}}=\frac{t_{Y R} t_{Y L} e^{\gamma_{Y, 1} \Delta L_{1}}\left(m y_{11} m y_{22}-m y_{12} m y_{21}\right)}{m y_{22}+m y_{21} r_{Y L} e^{2 \gamma_{Y, 1} \Delta L_{1}}-r_{Y R}\left(m y_{12}+m y_{11} r_{Y L} e^{2 \gamma_{Y, 1} \Delta L_{1}}\right)}
$$

$$
\frac{R_{1}}{F_{\text {in }}}=\frac{\left(m y_{11} r_{Y R}-m y_{21}\right) t_{Y L} e^{\gamma_{Y \cdot 1} \Delta L_{1}}}{m y_{22}-r_{Y R} m y_{21}+\left(m y_{21}-r_{Y R} m y_{11}\right) r_{Y L} e^{2 \gamma_{Y \cdot 1} \Delta L_{1}}}
$$

layer, the carrier induced index change, $\Delta n$, is approximated by

$$
\Delta n=-\Gamma_{Y} \frac{\alpha_{m} \lambda_{Y}}{4 \pi} \frac{\partial G}{\partial N} \Delta N+\frac{\partial n}{\partial T} \Delta T
$$

where $\alpha_{m}$ is the linewidth enhancement factor, $\partial G / \partial N$ is the differential gain, $\partial n / \partial T$ is the temperature coefficient of refractive index, $\Delta T$ is the change of temperature and $\Delta N$ is the change of carrier concentration above threshold. $F_{1}$ and $R_{1}$ are the propagation fields at the interface between first and second matrix section.

The boundary conditions for the $Y$-polarized light at the right and left surfaces are given by

$$
\begin{aligned}
& R_{n}=r_{Y R} F_{n} \text { and } F_{\text {out }}=t_{Y R} F_{n} \\
& F_{1}=t_{Y L} e^{\gamma_{Y, 1} \Delta L_{1}} F_{\text {in }}+r_{Y L} e^{2 \gamma_{Y, 1} \Delta L_{1}} R_{1}
\end{aligned}
$$

where $r_{Y R(L)}$ and $t_{Y R(L)}$ is the reflection and transmission coefficient at the right (left) surface, respectively. Substitute the boundary conditions into $(\mathrm{C} 1)$ gives

$$
\begin{aligned}
& {\left[\begin{array}{ll}
m y_{11} & m y_{12} \\
m y_{21} & m y_{22}
\end{array}\right]^{-1}\left[\begin{array}{c}
F_{n} \\
r_{Y R} F_{n}
\end{array}\right]} \\
& \quad=\left[\begin{array}{cc}
r_{Y L} e^{2 \gamma_{Y, 1} \Delta L_{1}} & 0 \\
1 & 0
\end{array}\right]\left[\begin{array}{c}
R_{1} \\
0
\end{array}\right]+\left[\begin{array}{c}
t_{Y L} e^{\gamma_{Y, 1} \Delta L_{1}} F_{\text {in }} \\
0
\end{array}\right] .
\end{aligned}
$$

Using the fact that

$$
\left[\begin{array}{cc}
-1 & r_{Y L} e^{2 \gamma_{Y, 1} \Delta L_{1}} \\
0 & 0
\end{array}\right]\left[\begin{array}{cc}
r_{Y L} e^{2 \gamma_{Y, 1} \Delta L_{1}} & 0 \\
1 & 0
\end{array}\right]\left[\begin{array}{c}
R_{1} \\
0
\end{array}\right]=\left[\begin{array}{l}
0 \\
0
\end{array}\right]
$$

we have

$$
\begin{gathered}
{\left[\begin{array}{rc}
-1 & r_{Y L} e^{2 \gamma_{Y, 1} \Delta L_{1}} \\
0 & 0
\end{array}\right]\left[\begin{array}{ll}
m y_{11} & m y_{12} \\
m y_{21} & m y_{22}
\end{array}\right]^{-1}\left[\begin{array}{c}
F_{n} \\
r_{Y R} F_{n}
\end{array}\right]} \\
\quad=\left[\begin{array}{c}
-t_{Y L} e^{\gamma_{Y, 1} \Delta L_{1}} F_{\mathrm{in}} \\
0
\end{array}\right] .
\end{gathered}
$$

After further simplification and using the boundary condition $F_{\text {out }}=t_{Y R} F_{n}$, we have (C9) shown at the top of the page.

$R_{1}$ can also be expressed in term of $F_{\mathrm{in}}$. Using the similar transformation in $(\mathrm{C} 7)$, we can write

$$
\left[\begin{array}{cc}
-r_{Y R} & 1 \\
0 & 0
\end{array}\right]\left[\begin{array}{cc}
1 & 0 \\
r_{Y R} & 0
\end{array}\right]\left[\begin{array}{c}
F_{n} \\
0
\end{array}\right]=\left[\begin{array}{l}
0 \\
0
\end{array}\right]
$$

Substituting (C10) into (C1), we get

$$
\begin{aligned}
{\left[\begin{array}{l}
0 \\
0
\end{array}\right]=} & {\left[\begin{array}{cc}
-r_{Y R} & 1 \\
0 & 0
\end{array}\right]\left[\begin{array}{ll}
m y_{11} & m y_{12} \\
m y_{21} & m y_{22}
\end{array}\right] } \\
& \cdot\left[\begin{array}{c}
r_{Y L} e^{2 \gamma_{Y, 1} \Delta L_{1}} R_{1}+t_{Y L} e^{\gamma_{Y, 1} \Delta L_{1}} F_{\text {in }} \\
R_{1}
\end{array}\right] .
\end{aligned}
$$

After further simplification, the expression of $R_{1} / F_{\text {in }}$ is given by (C12) shown at the top of the page.

The photon density inside the $k$ th dielectric layer can be expressed as

$$
\begin{aligned}
{\left[\begin{array}{l}
F_{k} \\
R_{k}
\end{array}\right]=} & \prod_{j=1}^{k-1}\left[\begin{array}{cc}
e^{\gamma, j+1} \Delta L_{j+1} & 0 \\
0 & e^{-\gamma_{Y, j+1} \Delta L_{j+1}}
\end{array}\right] \\
& \cdot\left[\begin{array}{ll}
\frac{n y_{j+1}+n y_{j}}{2 n y_{j+1}} & \frac{n y_{j+1}-n y_{j}}{2 n y_{j+1}} \\
\frac{n y_{j+1}-n y_{j}}{2 n y_{j+1}} & \frac{n y_{j+1}+n y_{j}}{2 n y_{j+1}}
\end{array}\right]\left[\begin{array}{l}
F_{1} \\
R_{1}
\end{array}\right] \\
= & {\left[\begin{array}{ll}
p_{11} & p_{12} \\
p_{21} & p_{22}
\end{array}\right]\left[\begin{array}{l}
F_{1} \\
R_{1}
\end{array}\right] . }
\end{aligned}
$$

Using the boundary conditions, $F_{k}$ and $R_{k}$ can be written as

$$
\begin{aligned}
& F_{k}=\left(r_{Y L} e^{2 \gamma_{Y, 1} \Delta L_{1}} p_{11}+p_{12}\right) R_{1}+p_{11} t_{Y L} e^{\gamma_{Y, 1} \Delta L_{1}} F_{\text {in }}, \\
& R_{k}=\left(r_{Y L} e^{2 \gamma_{Y, 1} \Delta L_{1}} p_{21}+p_{22}\right) R_{1}+p_{21} t_{Y L} e^{\gamma_{Y, 1} \Delta L_{1}} F_{\text {in }} .
\end{aligned}
$$

\section{REFERENCES}

[1] Y. Suematsu, K. Iga, and S. Arai, "Advanced Semiconductor Lasers," Proc. IEEE, vol. 80, pp. 383-397, 1992.

[2] K. D. Chouette and R. E. Leibenguth, "Control of vertical cavity laser polarization with anisotropic transverse cavity geometries," IEEE Photon. Technol. Lett., vol. 6, pp 40-42, Jan. 1994.

[3] T. Mukaihara, F. Koyama, and K. Iga, "Engineered polarization control of GaAs/AlGaAs surface emitting lasers by anisotropic stress from elliptical etched substrate hole," IEEE Photon. Technol. Lett., vol. 5, pp 133-135, Feb. 1993

[4] T. Numai, K. Kurihara, K. Kühn, H. Kosaka, I. Ogura, M. Kajita, H. Saito, and K. Kasahara, "Control of light-output polarization for surfaceemitting-laser type device by strained active layer grown on misoriented substrate," IEEE J. Quantum Electron., vol. 31, pp. 636-642, 1995.

[5] M. Shimizu, T. Mukaihara, F. Koyama and K. Iga, "A method of polarization stabilization in surface emitting lasers," Japan J. Appl. Phys., vol 30., pp. L1015-L1017, 1991.

[6] J. H. Ser, Y. G. Ju, J. H. Shin, and Y. H. Lee, "Polarization stabilization of vertical cavity top surface emitting lasers by inscription of fine metal interlaced gratings," Appl. Phys. Lett., vol. 66, pp. 2769-2771, 1995.

[7] F. Robert, P. Besnard, M.-L. Chares, and G.M. Stephan, "Switching of the polarization state of a vertical cavity surface emitting laser using polarized feedback," Optic. Quantum Electron., vol. 27, pp. 805-811, 1995. 
[8] H. Kawaguchi, "Absorptive and dispersive bistability in semiconductor injection lasers," Optic. Quantum Electron., vol. 19, pp. S1-S36, 1987.

[9] C. J. Chang-Hasnain, J. P. Harbison, G. Hasnain, A. C. Von Lehmen, L. T. Florez, and N. G. Stoffel, "Dynamic, polarization, and transverse mode characteristics of vertical cavity surface emitting lasers," IEEE $J$. Quantum Electron., vol. 27, pp. 1402-1409, 1991.

[10] F. Koyama, K. Morito, and K. Iga, "Intensity noise and polarization stability of GaAlAs-GaAs surface emitting lasers," IEEE J. Quantum Electron., vol. 27, pp. 1410-1416, 1991.

[11] K. D. Choquette, R.P. Schneider, Jr., Kevin, L. Lear, and R. E. Leibenguth, "Gain-dependent polarization properties of vertical cavity lasers," IEEE J. Select. Topics Quantum Electron., vol 1, pp. 661-666, 1995.

[12] S. F. Yu, W. N. Wong, P. Shum, and E. H. Li, "Theoretical analysis of modulation response and second harmonic distortion in vertical cavity surface emitting lasers," IEEE J. Quantum Electron., vol. 32, pp. 2139-2147, Dec. 1996.

[13] S. F. Yu, "Dynamic behavior of vertical cavity surface emitting lasers," IEEE J. Quantum Electron., vol. 32, p. 1168-1179, 1996

[14] G. Björk and O. Nilsson, "A new exact and efficient numerical matrix theory of complicated laser structures: Properties of asymmetric phaseshifted DFB lasers," J. Lightware Technol., vol. LT-5, pp. 140-146, Jan. 1987.

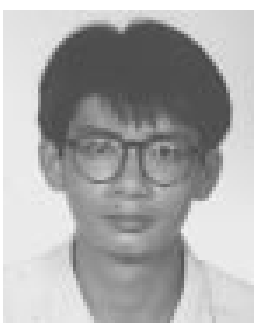

S. F. Yu received the B.Eng. degree (with Departmental Prize) in electronic engineering from University College London, London, England, in 1990 and the Ph.D. degree in optoelectronics from Cambridge University, Robinson College, Cambridge, England, in 1993.

In 1994, he joined the Department of Electrical and Electronic Engineering, University of Hong Kong, where he is currently a Lecturer. His main research topics include wavelength selectivity of grating coupled waveguides, transient properties of semiconductor laser diodes, and design of optoelectronic integrated circuit. $\mathrm{He}$ currently conducts the development of high-performance semiconductor lasers for the application of high-speed communication systems. He is also involved in the investigation of the influence of acousticwave in semiconductor materials.

Dr. Yu is a Fellow and Honorary Scholar of Cambridge Commonwealth Trust. He has held a Croucher Foundation Scholarship and an overseas research student award while studying for the doctoral program. 